Pecvnia, 2 (2006), pp. 65-78

\title{
Algunas consideraciones historiográficas sobre la lógica de la Partida Doble y la clasificación de Cuentas
}

\author{
J. Lanero Fernández \\ E. Ortega Montes
}

\begin{abstract}
El presente trabajo hace un recorrido historiográfico por diversos tratados contables con el propósito de ver la evolución de la clasificación de cuentas, sin perder de vista la lógica y sistemática que se deriva de la teneduría de libros por partida doble.

Todos los intentos de sistematizar y clasificar sirvieron para profundizar en la comprensión de la lógica y estructura de cuentas por el sistema de partida doble y para facilitar mejores métodos de exposición. El estudio rastrea diversos tratados, que se extienden en el tiempo, desde el siglo XVI hasta mediados del XIX.
\end{abstract}

Palabras clave: teneduría de libros, partida doble, clasificación de cuentas.
This paper analyzes various accounting books with a particular purpose in mind: checking the evolution of classification of accounts but taking into consideration the logic and method derived from the double-entry bookkeeping.

All the attempts at systematizing and classifying accounts served to deepen into the comprehension of the logic of the structure of accounts by means of the double-entry system and to pave the way for better methods of exposition. This study revises various accounting books spanning from $16^{\text {th }}$ Century to $19^{\text {th }}$ Century.

Key words: Bookkeeping, double-entry, classification of accounts. 
En The Universal Dictionary of Trade and Comerce, obra de Savary traducida al inglés, corregida y aumentada por Malachy Postlethwayt, que se publicó en Londres en 1751, hay un largo artículo que, bajo la entrada de "Mercantile Accountantship", entre otras cosas, señala:

In [the journal] the debitand credit are rationally fixed and settled, according to the principles of accountantship; and therefore, this requires the book-keeper's hand: (...) because in journalizing the waste book lies all the difficulty of account-keeping ${ }^{1}$.

De forma parecida, aunque con su estilo característico, se expresa Roger North, que es la "Person of Honour" que escribió The Gentleman Accomptant, publicado en 1714:

The marking true Drs. and Crs. is the greatest Difficulty of Accompting, and perpetually exerciseth the Judgement; being an Act of the Mind, intent upon the Nature and Truth of Things; for this Reason, the entring Matters formally in the Books, is a Business, that will not be done in haste, no more than calculating Propositions in Arithmetick and Geometry; and an Error of that kind spoils all. The best general Direction for avoiding false Charges, is to consider attentively the Consequence of every Entry, as if each Accompt were to go away with its Portion; and then to write so, that each shall have no more than its Due, and not one what is due to another. For no J usticiary in the World can be more considerative and careful, jus suum cuique tribuendo, than an Accomptant ought to be in placing his Parcels to their proper Drs. and Crs. ${ }^{2}$

En sintonía con lo expresado por estos dos autores, la mayoría de los tratados contables publicados hasta mediados del siglo

\footnotetext{
[Malachy Potlethwayt], The Universal Dictionary of Trade and Commerce, Translated from the French of the Celebrated Monsieur Savary, Inspector General of the Manufactures, for the King, at the Custom-house of Paris: With Large Additions and Improvements, Incorporated Throughout the Whole Work; Which More Particularly Accommodate the Same to the Trade and Navigation of These Kingdoms, and the Laws, Customs, and Usages, To Which All Traders Are Subject. By Malachy Postlethwayt, Esq., London: Printed for J ohn and Paul Knapton, in LudgateStreet, MDCCLI [1741], vol. II, p. 210.

2 [Roger North], The Gentleman Accomptant, or, An Essay to Unfold the Mystery of Accompts. By Way of Debtor and Creditor, Commonly Called Merchants Accompts, Applying the Same to the Concerns of the Nobility and Gentry of England... Done by a Person of Honour..., London: Printed for E. Curil..., 1714, pp. 13-14.
} 
XIX, dedican tanto espacio como ingenuidad a instruir al lector para que distinga, para cada clase de operación, la cuenta apropiada del Libro Mayor en la que hay que cargar o abonar. Un método habitual era presentar, de varias formas, una enumeración detallada de lo que había de hacerse para cada tipo de transacción; en ocasiones, precedida de unas normas generales más o menos elaboradas y que, en la mayoría de los casos, confundían más que aclaraban los aspectos que presentaba al lector. Alexander Malcolm, en A Treatise of Book-Keeping, or, Merchants Accounts; in the Italian Method of Debtor and Creditor, publicado en 1731, menciona estas formas de exposición al escribir sobre "our English Writers on this subject":

They reduce all other particular Instructions and Rules to certain Heads, and Branches of Business (which is so far right). And then, in the Way of Question and Answer, they shew what Accounts are to be made Debtors, and what Creditors, in the various Cases and Transactions that most commonly occur under each of those Heads.

Añade que semejante método, aunque parece sencillo y adaptado para el lector, en realidad resulta tedioso y oscuro. Y sostiene que "...a very few general Rules comprehend all those Cases, in such a Manner as to make the Sense and Reason of what's to be done much more clear and obvious"13.

El objetivo pues, de este estudio, es hacer un recorrido historiográfico por diversos tratados contables, con el fin de ver la evolución de la clasificación de cuentas, sin perder de vista la lógica y sistemática que se deriva de la teneduría de libros por partida doble.

II

La dificultad más importante para sistematizar estas normas que, en caso de que fueran satisfactorias, no sólo deberían ser una guía clara para sus usuarios, sino que también deberían reflejar la lógica y el fundamento del sistema de la partida doble, es la que, todavía hoy, confunde a los que se inician en el estudio de la contabilidad y conduce a

Alexander Malcolm, A Treatise of Book-Keeping, or, Merchants Accounts; in the Italian Method of Debtor and Creditor..., By Alexander Malcolm, London: Printed for J. Osborn and T. Longman, 1731. 
la ambigüedad a los redactores de los principales periódicos económicos. Desde el punto de vista del propietario de un negocio, si un cargo es favorable o desfavorable depende de la naturaleza de la cuenta en la que el cargo está registrado. Los principiantes en el estudio de la contabilidad, que tienen problemas para entender esta dificultad, se quedarían tranquilos si supieran que el Príncipe Maurice de Orange se encontró en la misma situación cuando, a finales del siglo XVI, estudió la teneduría de libros por partida doble con el afamado matemático y hombre de negocios Simon Stevin. En el diálogo que mantienen, recogido en el tratado de Stevin, Vorstelicke Bovckhovding op de Italiaensche wyse..., publicado en 1607, sobre "interrogantes en la teneduría de libros", el Príncipe pregunta: Las partidas en el Libro Mayor son de cargo y abono, ¿cuáles me benefician y cuáles me perjudican?. A ello Stevin responde: El cargo beneficia, porque Pedro, que me debe mucho, aumenta mi capital. También beneficia tener mucha pimienta en el almacén y mucho dinero en caja, que están en el Debe; el Haber provoca lo contrario ${ }^{4}$.

Cuando el Príncipe pregunta si existen excepciones, Stevin, en un inicio, responde que no se le ocurre ninguna, pero el Príncipe sigue sin comprenderlo. Un saldo positivo en la cuenta del Libro Mayor por clavo, representa una ventaja para el propietario, aunque sea un abono. Y da más ej emplos. Stevin lo reconoce y pasa a enunciar la regla de que el cargo es negativo y el abono favorable en todas las partidas de la cuenta de capital, o en otras cuentas que la representen, tal es el caso de la cuenta de pérdidas y ganancias. Ignoramos si el Príncipe se quedó satisfecho con la respuesta que recibió, dado que la deuda de capital significa lo mismo que si el patrón o propietario dijera: Yo, Nombre, soy deudor, cuanto más un hombre se endeuda, mayor es su desventaja; y cuanto más es acreedor, mayor es la ventaja, de tal forma que es lo contrario a otras cuentas del Libro Mayor ${ }^{5}$.

Con el fin de reducir la dificultad para el Príncipe, de tal suerte que tuviera sentido y razón lo que debía hacerse, exige una clasificación apropiada de las cuentas del Libro Mayor. La argumentación general de Stevin, en cierto modo, toma esa dirección, aunque la primera clasificación de cuentas que se presenta en un tratado contable iba a

\footnotetext{
4 Simon Stevin, Vorstelicke Bovckhovding op de Italiaensche wyse in domeine en finance extraordinaire..., Leyden: By lan Bouwensz, 1607. La traducción es nuestra.

5 Ibid.
} 
aparecer ochenta años después. Se debe a la autoría de un holandés del que, a parte de su libro, Kort begryp vant beschouwig onderwijs in t koopmans boekhouden..., publicado en 1681, nada más sabemos ${ }^{6}$.

III

El libro de Van Gezel se aparta radicalmente de la inmensa mayoría de los anteriores y contemporáneos, pues no incluye modelos de ejemplos o listas detalladas de tipos de operaciones. En su lugar, presenta un tratamiento analítico del tema, a nivel de aplicación, con la promesa de que, con la ayuda de Dios, se compromete a publicar otras obras con ejemplos y aplicaciones prácticas. Su originalidad es manifiesta, aunque se aprecia cierta influencia de Stevin, cuya obra conocía.

En el nivel más general de clasificación, para Van Gezel tan solo existen dos tipos de cuentas: las "propias" (eigene) y las de "otros" o "contrarios" (tegengestelde). Una cuenta propia es la que su cargo significa la deuda del mercader o propietario, y su abono el crédito del mercader. Esta explicación, a todas luces críptica, se amplía inmediatamente. Las cuentas propias se llevan de tal forma que el propietario pueda saber cuál es su capital inicial (propiedad), y por qué y cuánto aumenta o disminuye en determinado período comercial. Por otra parte, las cuentas de los contrarios se necesitan para que muestren en qué situación se encuentra el propietario con cada persona, el dinero y las mercancías con las que comercia. Las cuentas "propias" y de los "contrarios" son necesarias para suministrar la prueba necesaria a la hora de confeccionar la cuenta de balance. Luego se plantean las reglas para cargar o abonar en las cuentas del Libro Mayor. El autor distingue cuatro categorías de operaciones (posten): Las beneficiosas o wordeelig, las perjudiciales 0 nadeelind, las indiferentes o middlematig y las mixtas o vermengde.

Las operaciones de la primera categoría son las que incrementan el capital del propietario; las segundas lo disminuyen y las terceras no lo modifican. Se deduce que para una operación beneficiosa se abona en una cuenta propia y se carga en una contraria; lo opuesto es de aplicación en una operación perjudicial. En el caso de una operación indiferente, sólo afecta a las cuentas contrarias.

6 Willem Van Gezel, Kort begryp van't beschouwig onderwijs in 't koopmans boekhouden..., Amsterdam: By lan Ten Hoorn, 1681. 
Algunas consideraciones historiográficas sobre la lógica de la Partida Dobley la clasificación de Cuentas

Giovanni Antonio Moschetti, en su Dell'universal trattato di libri doppii, publicado en 1610, se ocupa de la naturaleza de las operaciones que afectan a la cuenta de capital. Y lo hace de tal forma que parece existir un cierto vínculo con la obra de Simon Stevin. También está en la misma línea de Van Gezel, obra posterior a la suya y que sigue un enfoque independiente ${ }^{7}$.

A su vez, Andrea Zambelli, en II ragionato, aparecido en 1671, afirma que las cuentas del Libro Mayor deben pertenecer a una de las dos categorías: privadas, o privati, y abiertas o largas, aperti o longhi. En un párrafo, ciertamente enigmático, sugiere que tenía en mente una clasificación bipartita, que correspondería a las cuentas propias y contrarias de Van Gezel, aunque parece llegar por diferente camino. No obstante, sus intenciones se eclipsan por el hecho de que coloca la cuenta de caja entre las cuentas abiertas, y dice que la cuenta bancaria puede ser privada o abierta, "secondo il caso occorrente"18.

En sus reglas para anotar los cargos y abonos, Van Gezel destaca la naturaleza de las cuentas y no, como era costumbre, el tipo de operación. Después de la publicación de su libro, que sólo conoció una edición, los tratados contables posteriores se centraron más en la clasificación de cuentas, aunque hasta el siglo XIX no se superó la planteada por Van Gezel.

Por otra parte, se producen ciertos intentos de hacer una exposición "teórica" del sistema de la partida doble. El primer ejemplo inglés de tal exposición analítica la encontramos en la obra de Hustcraft Stephen, Italian Book-Keeping Reduced into an Art, publicada en 1735 que, en parte, se ocupa de los mismos aspectos que contempla Van Gezel ${ }^{9}$.

Appresso Luca Valentini, 1610

8 Andrea Zambelli, II ragionato, o sia Trattato della scrittvra vniversale.., Milano: Per Federico Agnelli Scultore, \& Stampatore, 1671.

9 Hustcraft Stephens, Italian Book-Keeping Reduced into an Art: Being An Entire New and Compleat System of Accompts in General. Demonstrated in a Chain of Consequences from Clear and Self-evident Principles..., London: Printed for Mears, 1735. Véase también, B. S. Yamey, H. C. Edey and Hugh W. Thomson, eds., Accounting in England and Scotland: 1543-1800. Double Entry in Exposition and Practice, London: Sweet \& Maxwell, 1963, pp. 135-142; Harold Edey and B. S. Yamey, eds., Debits, Credits, Finance and Profits, London: Sweet \& Maxwell, 1974. 
IV

La clasificación bipartita de Van Gezel no fue ampliamente aceptada hasta bien entrado el siglo XIX. Por otro lado, la clasificación tripartita que se atribuye a otro holandés, Matthieu de la Porte, suscitó más simpatías, aunque resulta difícil medir su grado de influencia. Presentó su clasificación en La Science des Negocians et teneurs de livres, que se publicó por primera vez en 1704, aunque tuvo múltiples reediciones, traducciones y adaptaciones ${ }^{10}$.

De la Porte divide las cuentas del Libro Mayor en tres clases: las cuentas del propietario, le chef ou le négociant lui-même; las cuentas de activos y pasivos "naturales", les effets en nature; y las cuentas de las personas con las que comercia el propietario, les correspondans. La primera categoría incluye la cuenta de capital y la cuenta de pérdidas y ganancias con sus subdivisiones. La segunda, el dinero, la mercancía, letras a cobrar y pagar, barcos, casas, etcétera; y la tercera consta de deudores y acreedores. La exposición de cómo deben abordarse varios tipos de operaciones está dispuesta a modo de clasificación de cuentas.

De la Porte es proclive a las tricotomías y tríadas. En sus introductorias "Remarques sur les comptes en général", relaciona diez conceptos tripartitos, que incluyen las tres "sortes de négociations", comprar, vender y el trueque; y las tres formas de concluir las cuentas: con beneficios, pérdidas o sin una cosa ni la otra. La clasificación de las cuentas es una de sus tríadas.

El sistema seguido por De la Porte lo adaptó directamente Pietro Scali en su obra Trattato del modo di tenere la scrittura dei mercanti a partite dopie, publicada en 1755. Lamentablemente, el enfoque es confuso, pues Scali parece haber interpretado, de forma inadecuada, la presentación tabular que hallamos en el tratado de de la Porte ${ }^{11}$.

10 Matthieu de La Porte, La science des negocians et teneurs de livres, ou instruction generale pour tout ce qui se pratique dans les comptoirs des negocians, tant pour les affaires de Banque, que pour les marchandises; \& chez les financiers pour les comptes..., Paris: Chez Guillaume Cavelier, Charles Osmont, 1704.

11 Prieto Scali, Trattato del modo di tenere la scrittura dei mercanti a partite dopie cioè all'italiana e descrizione del bilancio della prima e della seconda regione, composto da Pietro Paolo Scali dedicato agl'Illustriss. Signori Mercanti in Livorno, Livorno: Nella Stamperia di Gio. Paolo Fantechi e Compagni., 1755. 
Una clasificación tripartita semejante la encontramos en un tratado anterior al de la Porte, obra de otro holandés, Abraham de Graaf, Instructie van het Italiaans boeckhouden, publicada en 1688. Las diferencias entre los dos sistemas de clasificación difieren, tan sólo, en detalles. Da la impresión que de Graaf y de la Porte llegaron a sus esquemas de clasificación de forma independiente, aunque no debemos descartar que ambos se inspirasen en una fuente común ${ }^{12}$.

Una clasificación tripartita similar se convirtió en habitual en los tratados ingleses del siglo XVIII y primeras décadas del XIX. Además de las cuentas personales y reales, los tratados mencionan una tercera categoría: las cuentas "ficticias" o "imaginarias", que corresponden a la cuenta del propietario en de la Porte. Una definición típica de las cuentas imaginarias es la que podemos observar en uno de los primeros tratados americanos. Se trata del libro de J ames Bennet, The American System of Practical Book-Keeping, publicado en Nueva York en 1820:

Imaginary Accounts are fictious titles invented to represent the merchant himself, or to supply the want of real or personal titles, in recording such gains or losses as cannot be ascribed, or with propriety placed to real or personal accounts ${ }^{13}$. ingleses anteriores ${ }^{14}$.

El Profesor Yamey, por su parte, documenta ejemplos

A comienzos del siglo XIX, pues, esta clasificación tripartita había pasado a ser corriente en los tratados ingleses. F.W. Cronhelm le dedica una crítica incisiva en su libro de 1818, Double Entry by Single, llegándola a definir como "one of the most ludicrous that ever enlivened the gravity of the scientific page". Su nomenclatura le resulta torpe, aunque, sobre todo, refleja una ignorancia del "great essential principle of bookkeeping"; es decir: el principio de igualdad entre el valor de "the whole

12 Abraham de Graaf, Instructie van het Italiaans boeckhouden, mete en Memorial toegepaast op de Negotie Particulier, in Commissie, en in Compagnie. Door Abraham de Graff, Amsterdam: By de Weduwe van Gysbert de Groot, 1693. El Profesor Yamey sostiene que existe, incluso, una edición anterior a la de 1688. Cfr. Harold Edey and B.S. Yamey, eds., Debits, Credits, Finance and Profits, ed. cit., p. 157.

13 James Bennett, The American System of Practical Book-Keeping, Adapted to the Commerce of the United States, in Its Domestic and Foreign Relations; and Exemplified in One Set of Books, Kept by Double Entry... By J ames Bennett, New York: Printed by Abm. Paul, 1820, p. x.

14 Cfr. B.S. Yamey, H.C. Edey and Hugo W. Thomson, eds., Accounting in England and Scotland: 1543-1800. Double Entry in Exposition and Practice, ed. cit., pp. 57-58; 62, 65-67. 
capital", bien en el almacén del propietario, bien en su cuenta de capital, y la "sum of all its [capital's] parts", en el resto de las cuentas, "whatever variations they undergo, and whether the whole capital increase, diminish, or remain stationary". Desde este "clear and simple principle of the equality of the whole to the sum of its parts", del que erróneamente dice que "[had] never before been laid down as the basis for Book-Keeping", Cronhelm propone una clasificación bipartita de las cuentas: "Parts of Property", subdivididas en "personal", "money" y "merchandise"; y "whole Property", la cuenta de existencias con sus ramificaciones, como la cuenta de pérdidas y ganancias. Esto supone un regreso al esquema planteado por Van Gezel de cuentas propias y contrarias ${ }^{15}$.

Una clasificación bipartita similar de las cuentas en "whole property" y "parts of property" puede encontrarse en el tratado de C. Morrison, A Complete System of Practical Bookkeeping, que se publicó en $1815^{16}$.

\section{V}

Entre tanto, se había presentado otro esquema distinto de clasificación bipartita. Se debe a Bertrand François Barrême, cuyo libro Traite des parties doubles, publicado en París en 1721, estaba terminado antes de la muerte de su autor en 1703. Barrême enuncia la división de todas las cuentas del Libro Mayor en dos clases: Ias cuentas generales (les comptes généraux) y las cuentas particulares (les comptes particuliers) que abre para cada uno de sus deudores y acreedores (sus Correspondans) ${ }^{17}$. El término comptes géneraux, para abarcar todas las cuentas, salvo las personales, lo utiliza también Samuel Ricard en su tratado L'Art de bien

15 F.W. Cronhelm, Double Entry by Single, A New Method of Book-Keeping Applicable to All Kinds of Business; and Exemplified in Five Sets of Books, London: Longman, Hurst, Rees, Orme, and Brown, 1818.

16 C. Morrison, A Complete System of Practical Bookkeeping, Applicable to All Kinds of Business; Exemplified in Four Sets of Books, Individual and Partnership Concerns; Arranged by Single Entry, Double Entry in Present Practice... by C. Morrison, Third Edition, Glasgow: Printed by R. Chapman for Reid \& Henderson, 1822. Véase también Andreas Wagner, Neues Vollständiges und allgemeines Lehrbuch des Buchhaltens..., Entworfen und nach eignen Grundsätzen bearbeitet von Andreas Wagner, Magdeburg: bey Georg Christian Keil, 1802.

17 Bertrand François Barrême, Traite des parties doubles, ou méthode aisée pour apprendre à tenir en parties doubles les livres du commerce $\&$ des finances..., Paris: JeanMichel Garnier pour J ean Geofrey Nyon, 1721. 
tenir les livres de comptes, publicado en $1709^{18}$. Ya en 1540, Domenico Manzoni había dividido las cuentas en dos grupos: vivas, vive, y muertas, morte. Las primeras son todas aquellas que se refieren a "ogni creatura animata", y las segundas a "robbe, ouer ogni altra cosa". No obstante, esta división de Manzoni únicamente pretendía la disposición de las partidas en el índice del Libro Mayor: las cuentas vivas se anotaban en la parte derecha y las muertas en la izquierda del Libro' ${ }^{19}$.

Pero volvamos a Barrême. Su división no tiene fundamento en lo que toca a la estructura lógica de un conjunto de cuentas por partida doble, aunque pueda ser sensata para la división del Libro Mayor atendiendo a razones administrativas ${ }^{20}$. De ahí que, en su exposición de reglas para cargar y abonar en las cuentas, recurre a su personificación. De este modo, se refiere a la cuenta de caja de la siguiente manera: caja es un cajero al que el Capital ha conferido la gestión de su dinero ${ }^{21}$.

La influencia de Barrême la podemos observar en la obra de Edmon Dégrange que, a su vez, expandió la clasificación de cuentas con su libro La tenue des livres rendue facile, editado en París en 1795 y que tuvo un éxito notable ${ }^{22}$ debido, al menos en parte, a su contribución al desarrollo de los sistemas de cuentas en columna.

Dégrange comienza explicando que en el sistema de cuentas por partida simple, el Libro Mayor se circunscribía a las cuentas de las personas con las que se comerciaba, aunque señala que algunos mercaderes también incluían una cuenta de caja. Prosigue diciendo que la teneduría de libros por partida doble, durante mucho tiempo, pareció ser ininteligible

18 Samuel Ricard, L'art de bien tenir les livres de comptes en parties doubles a l'italienne avec une table de l'explication des termes..., Par Samuel Ricard, Amsterdam: Ches Paul Marret, 1709.

19 Domenico Manzoni, Qvaderno doppio col svo giornale, novamente composto, $\&$ diligentissimamente ordinato, secondo il costume di Venetia. Opera a ogni persona vtilissima, $\&$ molto neccesaria di Domenico Manzoni opitergiense. Cvm gratia el privilegio del Illustrissimo Senato di Venetia, per Anni diece, 1540, Parte I, capitulo XIII.

20 Los primeros tratados alemanes, los de Schreiber (1518) y Gotlieb (1531), dividen el Libro Mayor en dos libros independientes: uno para las cuentas de mercaderías y el otro para el resto. Esta división no contiene ningún significado pedagógico; tan sólo refleja una conveniencia práctica.

21 Para la personificación de cuentas en los tratados contables ingleses véase J. G. C. Jackson, "The History of Methods of Exposition of Double-Entry Book-Keeping in England" in A. C. Littleton and B.S. Yamey, eds., Studies in the History of Accounting, London: Sweet $\&$ Maxwell Limited, 1956, pp. 288-312.

22 J.H. Vlaemminck, Historia y doctrinas de la contabilidad. Versión española revisada y ampliada por J osé M. González Ferrando, Madrid: Editorial E.J .E. S., 1961. 
y confusa, pues no se fundamenta en ninguna regla concreta y fija. Según Dégrange, la partida doble se diferencia de la simple en que la primera incluye las cuentas generales que representan al mercader. Divide estas cuentas generales en cinco categorías, las "cinq comptes généraux" que corresponden a los "cinq objets principaux", que sirven al comercio de "moyens d'échange". Estas cinco categorías son las cuentas de mercaderías, de dinero, de letras a cobrar, de letras a pagar y de pérdidas y ganancias. Estas cuentas generales deben considerarse como las del mercader: un cargo en una cuenta general debe considerarse como un débito al propio mercader bajo el nombre de la cuenta particular ${ }^{23}$. La idea de que las cuentas generales representan al propietario del negocio, aunque habitualmente se atribuye a Dégrange, apareció varias décadas antes en la obra de Pierre Giraudeau, La Banque rendue facile, publicada en 1741. Quizá Dégrange la presentó de un modo más explícito y accesible ${ }^{24}$.

La quíntuple división de las cuentas generales que realiza Dégrange es la base de mucha literatura contable posterior ${ }^{25}$. La bienvenida más calurosa se la dieron los denominados Cinque contisti italianos. Los más extremistas limitaron el número de cuentas generales del Libro Mayor a cinco, aunque Dégrange, en el esquema que presenta, permite posteriores subdivisiones para cada una de las cinco categorías.

La cuestión de la clasificación de cuentas, casi siempre dentro del marco de una teoría de la estructura de un sistema de cuentas, continuó ocupando a los especialistas del siglo XIX. Algunas ideas, ciertamente antiguas, se presentaron como novedades. Se abría así el campo para nuevas teorías, viej as especulaciones y constantes controversias. Sin embargo, nos inclinamos a pensar que la mayor parte de la docencia, en la práctica, se asentó en inculcar a los alumnos las reglas de cargar y

23 Edmon Dégrange, La tenue de livres rendue facile, ou nouvelle méthode d'enseignement, à l'usage des personnes destinées au commerce, avec une nouvelle méthode pour tenir des livres en double partie, par le moyen d'un seul registre dont tous les comptes balancent journellement. Sixième édition, revue, cossignée et augmentée, Paris: Hocquart \& Bordeaux, chez l'auteur et chez Filiatre [\&] de l'imprimerie de J. Foulquier, 1806, pp. 5-9.

24 Pierre Giraudeau, La Banque rendue facile aux principales nations de I'Europe, Genève: Chez l'auteur, 1741.

25 E. Peragallo, Origin and Evolution of Double Entry Bookkeeping; A Study of Italian Practice from the Fourteenth Century, New York, 1938, p. 100. 
Algunas consideraciones historiográficas sobre la lógica de la Partida Dobley la clasificación de Cuentas

abonar en las cuentas del Libro Mayor, sin encomendarles nada más que la obtención de resultados.

Desde una perspectiva más amplia, todos estos intentos de sistematizar y clasificar sirvieron para profundizar en la comprensión de la lógica de la estructura de cuentas por el sistema de partida doble, y para facilitar mejores métodos de exposición. A estos intentos se remonta el desarrollo de una serie de conjuros nemotécnicos, reglas y razonamientos que, se supone, ayudan al principiante a dar sus primeros pasos en los misterios de la partida doble. Puede que una de las más sorprendentes descripciones de la misma la diera J ohan Gottlieb en la lejana fecha de 1531. Llama poderosamente la atención su explicación de por qué las deudas que se contraen con terceras personas deben anotarse en la parte derecha del Libro Mayor: se trata, según él, de un proceso natural porque la verdad y la fe se representan siempre con la mano derecha. Puede que convenciera a sus factores, puede, pero la argumentación, a lo sumo, no pasa de ser ingeniosa.

\section{BIBLIOGRAFÍA}

BARRÊME, Bertrand François (1721) Traite des parties doubles, ou méthode aisée pour apprendre à tenir en parties doubles les livres du commerce \& des finances... Paris: Jean-Michel Garnier pour Jean Geofrey Nyon.

BenNeTt, James (1820) The American System of Practical Book-Keeping, Adapted to the Commerce of the United States, in Its Domestic and Foreign Relations; and Exemplified in One Set of Books, Kept by Double Entry... New York: Printed by Abm. Paul.

Cronhelm, F.W. (1818) Double Entry by Single, A New Method of BookKeeping Applicable to All Kinds of Business; and Exemplified in Five Sets of Books. London: Longman, Hurst, Rees, Orme, and Brown.

DE GRAAF, Abraham (1693) Instructie van het Italiaans boeckhouden, mete en Memorial toegepaast op de Negotie Particulier, in Commissie, en in Compagnie. Amsterdam: By de Weduwe van Gysbert de Groot.

DE LA PORTE, Matthieu (1704) La science des negocians et teneurs de livres, ou instruction generale pour tout ce qui se pratique dans les comptoirs des negocians, tant pour les affaires de Banque, que pour les marchandises; $\&$ chez les financiers pour les comptes... Paris: Chez Guillaume Cavelier, Charles Osmont. 
DÉGRANGE, Edmon (1806) La tenue de livres rendue facile, ou nouvelle méthode d'enseignement, à l'usage des personnes destinées au commerce, avec une nouvelle méthode pour tenir des livres en double partie, par le moyen d'un seul registre dont tous les comptes balancent journellement. Sixième édition, revue, cossignée et augmentée, Paris: Hocquart \& Bordeaux, chez l'auteur et chez Filiatre [\&] de l'imprimerie de J. Foulquier.

EdEY, Harold and B.S. YAMEY, eds. (1974) Debits, Credits, Finance and Profits. London: Sweet \& Maxwell.

GIRAUDEAU, Pierre (1741) La Banque rendue facile aux principales nations de I'Europe. Genève: Chez l'auteur.

JACKSON, J.G.C. (1956) "The History of Methods of Exposition of DoubleEntry Book-Keeping in England" in A. C. Littleton and B.S. Yamey, eds., Studies in the History of Accounting. London: Sweet \& Maxwell Limited.

MALCOLM, Alexander (1731) A Treatise of Book-Keeping, or, Merchants Accounts; in the Italian Method of Debtor and Creditor... London: Printed for J. Osborn and T. Longman.

MANZONI, Domenico (1540) Qvaderno doppio col svo giornale, novamente composto, \& diligentissimamente ordinato, secondo il costume di Venetia. Opera a ogni persona vtilissima, \& molto neccesaria di Domenico Manzoni opitergiense. Cvm gratia el privilegio del Illustrissimo Senato di Venetia, per Anni diece.

MORRISON, C. (1822) A Complete System of Practical Bookkeeping, Applicable to All Kinds of Business; Exemplified in Four Sets of Books, Individual and Partnership Concerns; Arranged by Single Entry, Double Entry in Present Practice... Third Edition, Glasgow: Printed by R. Chapman for Reid \& Henderson.

MoschetTI, Giovanni Antonio (1610) Dell'universal trattato di libri doppii... Venetia: Appresso Luca Valentini.

NORTH, Roger (1714) The Gentleman Accomptant, or, An Essay to Unfold the Mystery of Accompts. By Way of Debtor and Creditor, Commonly Called Merchants Accompts, Applying the Same to the Concerns of the Nobility and Gentry of England... Done by a Person of Honour... London: Printed for E. Curil...

Peragallo, E. (1938) Origin and Evolution of Double Entry Bookkeeping; A Study of Italian Practice from the Fourteenth Century. New York: American Institute Pub. Co. 
POTLETHWAYT, Malachy (1741) The Universal Dictionary of Trade and Commerce, Translated from the French of the Celebrated Monsieur Savary, Inspector General of the Manufactures, for the King, at the Custom-house of Paris: With Large Additions and Improvements, Incorporated Throughout the Whole Work; Which More Particularly Accommodate the Same to the Trade and Navigation of These Kingdoms, and the Laws, Customs, and Usages, To Which All Traders Are Subject. London: Printed for J ohn and Paul Knapton.

RICARD, Samuel (1709) L'art de bien tenir les livres de comptes en parties doubles a l'italienne avec une table de l'explication des termes..., Par Samuel Ricard. Amsterdam: Ches Paul Marret.

SCALI, Prieto (1755) Trattato del modo di tenere la scrittura dei mercanti a partite dopie cioè all'italiana e descrizione del bilancio della prima e della seconda regione, composto da Pietro Paolo Scali dedicato agl'lllustriss. Signori Mercanti in Livorno, Livorno: Nella Stamperia di Gio. Paolo Fantechi e Compagni.

STEPHENS, Hustcraft (1735) Italian Book-Keeping Reduced into an Art: Being An Entire New and Compleat System of Accompts in General. Demonstrated in a Chain of Consequences from Clear and Self-evident Principles... London: Printed for Mears.

STEVIN, Simon (1607) Vorstelicke Bovckhovding op de Italiaensche wyse in domeine en finance extraordinaire... Leyden: By lan Bouwensz.

VAN GEZEL, Willem (1681) Kort begryp van't beschouwig onderwijs in 't koopmans boekhouden... Amsterdam: By Ian Ten Hoorn.

VlaEMMINCK, J.H. (1961) Historia y doctrinas de la contabilidad. Versión española revisada y ampliada por J osé M. González Ferrando. Madrid: Editorial E.J.E.S.

WAGNER, Andreas (1802) Neues Vollständiges und allgemeines Lehrbuch des Buchhaltens..., Entworfen und nach eignen Grundsätzen bearbeitet von Andreas Wagner. Magdeburg: bey Georg Christian Keil.

YAMEY, B.S.; H.C. EdEY and Hugh W. ThOMSON, eds. (1963) Accounting in England and Scotland: 1543-1800. Double Entry in Exposition and Practice. London: Sweet \& Maxwell.

ZAMBeLLI, Andrea (1671) II ragionato, o sia Trattato della scrittvra vniversale... Milano: Per Federico Agnelli Scultore, \& Stampatore. 